\title{
ANÁLISIS TERMODINÁMICO DE UN CHILLER DE ABSORCIÓN DE 1 Y 2 ETAPAS DE UNA PLANTA DE COGENERACIÓN
}

\author{
THERMODYNAMIC ANALYSIS OF ONE AND \\ TWO STAGES ABSORPTION CHILLER POWERED \\ BY A COGENERATION PLANT
}

\author{
Hugo F. Zúñiga-Puebla ${ }^{1, *}$, E.C. Vallejo-Coral ${ }^{2}$, José Ramón Vega Galaz ${ }^{3}$
}

\section{Resumen}

Se han desarrollado modelos termodinámicos de enfriadores de agua por absorción de una etapa y ciclo no común de dos etapas que usan calor de desecho (de motores de combustión interna de 8,7 MW cada uno) para analizar las condiciones de operación de los equipos. Se ha realizado la comparación del sistema de una etapa con el sistema propuesto (2 etapas) en esta investigación. El coeficiente de desempeño (COP) obtenido para ambos sistemas fue el mismo, pero el calor removido del espacio refrigerado aumento de 1,3 MW (una etapa) a 1,6 MW (dos etapas) debido a que se recupera más energía residual utilizando un segundo generador. El calor residual aprovechado por el equipo de refrigeración fue de $3,8 \mathrm{MW}$ y el factor de planta del proceso de cogeneración fue de $58,11 \%$ y la capacidad de refrigeración del equipo fue de 1,623 kW. Finalmente, los ahorros económicos estimados por concepto de energía eléctrica que se tienen por poner en marcha el sistema de refrigeración por absorción que utiliza gases de escape como fuente de energía en lugar de un equipo común de refrigeración por compresión de la misma capacidad son 142 000,00 USD/año.

Palabras clave: absorción, calor recuperado, cogeneración, COP, energía desperdiciada

\begin{abstract}
Thermodynamics models of a single and a noncommon double stage ammonia-water absorption chiller that use waste heat (from three reciprocating engines of 8.7 MW each one) are developed to analyze the performance of the chiller for different operative conditions. A comparison of a single stage refrigeration system with the two stages proposed system is performed in this paper. The coefficient of performance (COP) obtained for both systems are the same, but the heat flux removed from the cooling media with the two-stage system increase from 1.3MW (single stage) until 1.6 MW due to the heat recovered increased with the second generator. The heat recovered used by the chiller was $3.8 \mathrm{MW}$, and the utilization factor of the cogeneration plant was $58.11 \%$, and the cooling capacity of the equipment was $1,623 \mathrm{~kW}$. Finally, the estimated economics savings for electric power due to the implementation of the absorption chiller that uses exhaust gases in place of a common refrigeration system by vapor compression with the same cooling capacity was $142,000.00$ USD/year.
\end{abstract}

Keywords: Absorption chiller, cogeneration, heat recovery refrigeration, waste energy, COP

\footnotetext{
1,*Facultad de Ingeniería Mecánica, Escuela Politécnica Nacional, Ecuador. Autor para correspondencia hugo.zuniga@epn.edu.ec (D) http://orcid.org/0000-0003-0732-8108.

${ }^{2}$ Instituto Nacional de Eficiencia Energética y Energías Renovables, Ecuador. (D) http://orcid.org/0000-0003-2065-0484.

${ }^{3}$ Departamento de Ingeniería Eléctrica, Instituto Tecnológico y de Estudios Superiores de Monterrey ITESM, México (D) http://orcid.org/0000-0003-7630-2035.
}

Recibido: 14-03-2018, aprobado tras revisión: 03-12-2018

Forma sugerida de citación: Zúñiga-Puebla, H. F.; Vallejo Coral, E. C. y Vega Galaz, J. R. (2019). «Análisis termodinámico de un chiller de absorción de 1 y 2 etapas de una planta de cogeneración». InGENiUs. N. ${ }^{\circ} 21$, (enerojunio). pp. 41-52. DOI: https://doi.org/10.17163/ings.n21.2019.04. 


\section{Introducción}

Los sistemas de refrigeración por compresión de vapor son ampliamente utilizados en la industria de refrigeración. En años recientes, el escenario de demanda de energía para el enfriamiento industrial ha experimentado cambios significativos; uno de estos ha sido la prohibición del uso del clorofluorocarbono (CFC) como agente refrigerante debido a su efecto sobre la capa de ozono. Por lo tanto, se ha estudiado el uso de fluidos alternativos en sistemas de refrigeración por compresión y, actualmente, los fabricantes de estos equipos utilizan otro tipo de fluidos como, por ejemplo, el isobutano (R600a) [1].

Ahora, los sistemas de refrigeración por compresión presentan desventajas, entre estas se encuentran el alto consumo de electricidad, altos costos de operación y contaminación. Los sistemas de refrigeración por absorción, que son alimentados por calor en lugar de electricidad, podrían ser una buena alternativa para reducir el consumo de electricidad y obtener ganancias económicas [2-4].

Los sistemas de refrigeración por absorción (SRA) se desarrollaron para utilizar energía térmica en vez de electricidad como entrada del ciclo. De esta forma, los SRA de dos etapas se utilizan para mejorar la operación de estos sistemas $[2,5,6]$. Ambos sistemas trabajan con dos fluidos: uno refrigerante y otro que absorbe el refrigerante con el fin de incrementar la presión por medio de una bomba. Los pares más comunes de fluidos reportados en la literatura son bromuro de litio/agua $\left(\mathrm{H}_{2} \mathrm{O}\right.$ como refrigerante y $\mathrm{LiBr}$ como medio de transporte) y amoníaco/agua $\left(\mathrm{NH}_{3}\right.$ como refrigerante y $\mathrm{H}_{2} \mathrm{O}$ como medio de transporte). Los sistemas amoníaco/agua se utilizan para obtener bajas temperaturas de evaporación (por ejemplo, para aplicaciones de congelación) [7]. Por lo tanto, los SRA podrían ser una buena alternativa para reducir el consumo de electricidad en el enfriamiento industrial y obtener ganancias económicas [2-4].

Colorado y Rivera [6] compararon un sistema de refrigeración por compresión de vapor con un sistema híbrido (compresión/absorción) considerando la primera y segunda leyes de la termodinámica; ellos utilizaron R134a y $\mathrm{CO}_{2}$ como refrigerantes en el sistema de compresión, y una solución de $\mathrm{H}_{2} \mathrm{O} / \mathrm{LiBr}$ en el ciclo de absorción. El sistema híbrido tiene un intercambiador de calor cascada, donde el condensador del sistema de compresión es el evaporador del sistema de absorción. El objetivo principal fue reducir la energía utilizada en el compresor. Los resultados de la investigación muestran que el sistema híbrido consume un $45 \%$ menos de electricidad que el ciclo de compresión simple. Asimismo, el coeficiente de desempeño (COP) obtenido en el sistema híbrido es más alto con el R134a.
Una de las ventajas de los SRA es la posibilidad de utilizar varias fuentes de calor como entrada al generador. Said et al. [8] diseñaron y construyeron un equipo de absorción con $\mathrm{NH}_{3} / \mathrm{H}_{2} \mathrm{O}$ que funciona con energía solar, para las condiciones climáticas de Arabia Saudí. El objetivo del sistema era producir hielo y enfriar el salón principal de un edificio, un recinto cerrado que constituye una carga de refrigeración de $10 \mathrm{~kW}$. Durante la prueba, la capacidad más alta de refrigeración mostrada por el equipo fue $10,1 \mathrm{~kW}$, y produjo hielo a pesar de la alta temperatura de condensación $\left(45^{\circ} \mathrm{C}\right)$ debido a las condiciones climáticas de verano. Otros autores como Wang et al. [9] estudiaron las fuentes óptimas de calor para diferentes aplicaciones de SRA.

El calor desechado por los gases de escape de las calderas, motores de combustión y turbinas puede ser utilizado como una fuente de calor para un sistema de absorción. Du et al. [10] construyeron un prototipo de un sistema de refrigeración de amoníaco/agua de una sola etapa que utilizaba el calor desechado por un motor diésel con un método activo de tubería abierta de calentamiento, diseñado para obtener una cantidad uniforme de calor disponible. Los autores diseñaron el intercambiador de calor para recuperar la energía desechada para una capacidad específica, y combinaron los procesos de condensación y absorción en una unidad que enfriaba circulando una solución que había sido enfriada previamente.

El sistema tuvo un COP de 0,53, y una capacidad de enfriamiento de $33,8 \mathrm{~kW}$ para una temperatura de los gases disipados de $567{ }^{\circ} \mathrm{C}$.

En la actualidad, fabricantes como AGO están construyendo sistemas de refrigeración por absorción que pueden acoplarse con un intercambiador de calor para utilizar un colector solar o un regenerador de agua caliente por recuperación de calor (heat recovery hot water regenerator, HRHWG, por sus siglas en inglés) con energía de los gases de escape [11].

Este tipo de sistemas presentan diferentes ventajas comparadas con sistemas de generación de potencia; algunas de estas ventajas son: (i) eficiencia energética creciente con co/trigeneración, (ii) reducción de las emisiones de $\mathrm{CO}_{2}$, y (iii) sustitución de potencia eléctrica de alta calidad por energía térmica de baja calidad en la forma de calor desechado. Además, existen también motivaciones económicas como: reducción de los costos de operación al utilizar calor desechado como la fuente de energía, y uso mínimo de la electricidad.

El objetivo principal de este trabajo es desarrollar un modelo termodinámico de una etapa y un sistema AGO (no convencional) de dos etapas con un equipo de absorción de amoníaco/agua diseñado y construido por AGO [11] para analizar el desempeño de dicho equipo bajo diferentes condiciones de operación. El equipo de absorción utiliza energía recuperada de los gases de escape de motores de combustión interna. 


\subsection{Descripción del sistema completo de coge- neración}

La Figura 1 muestra un esquema de una planta de cogeneración de potencia (PCP), que fue instalada por la compañía «Unión Energética del Noroeste SA de $\mathrm{SV} »$ y que vende electricidad y es una sucursal de la compañía «Negocio Agrícola San Enrique SA de $\mathrm{SV} »$. Esta última utiliza equipos para refrigerar sus productos agrícolas. Ambas compañías están en Agua Prieta, en el estado de Sonora al noroeste de México.

La PCP tiene tres motores de combustión interna con una salida total de 26,1 MW (e) (cada motor es de $8,7 \mathrm{MW}$ (e) y $9,8 \mathrm{MW}$ (t) se recuperan de los gases de escape y del circuito de refrigeración de alta temperatura. Un generador de vapor por recuperación de calor (heat recovery steam generator, HRSG, por sus siglas en inglés) recuperó $6 \mathrm{MW}(\mathrm{t})$ produciendo vapor, y un regenerador de agua caliente por recuperación de calor recuperó $3,8 \mathrm{MW}(\mathrm{t})$.

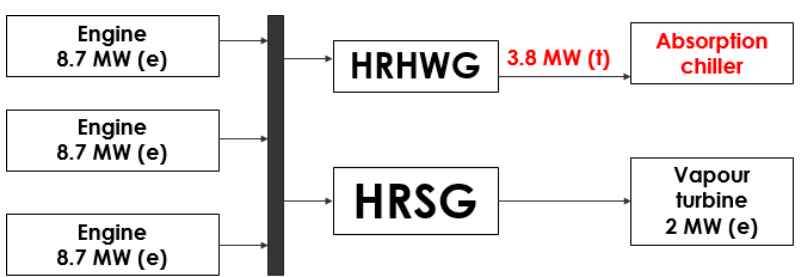

Figura 1. Planta de cogeneración de potencia bajo estudio.

El vapor producido con el HRSG se utiliza en una turbina de vapor para producir $2 \mathrm{MW}$ (e). El agua caliente producida en el HRHWG se utiliza como entrada a los equipos de absorción para producir aire frío para la refrigeración de almacenes donde se conservan los productos del área calurosa de Sonora.

\section{Fundamentos teóricos}

\subsection{Compresión versus absorción}

Se requiere energía para transportar calor desde un bulbo de baja temperatura a otro de alta temperatura. Un fluido refrigerante experimenta una serie de transformaciones termodinámicas, y cada refrigerante tiene un comportamiento único y definido, y en la medida en que dicho comportamiento es conocido, los ciclos impiden que sea necesario el reabastecimiento continuo de un refrigerante [12]. Los métodos principales de enfriamiento industrial son compresión y absorción.

El ciclo de compresión está basado en el ciclo de refrigeración convencional, en donde se transfiere calor desde el espacio que se está enfriando hacia el ambiente, utilizando energía eléctrica. El sistema de compresión tiene un condensador (unidad exterior), un evaporador (dentro del espacio que se está enfriando), una válvula de expansión y un compresor (que transporta el refrigerante) [13]. El desempeño del sistema es alto considerando que su entrada es energía eléctrica y su salida es calor que se remueve del evaporador. Estos dispositivos trabajan incrementando y reduciendo la presión del refrigerante, cambiando su temperatura de saturación.

El ciclo de absorción utiliza una bomba y no un compresor para incrementar la presión del sistema, lo cual reduce su consumo de electricidad. Sin embargo, requiere un flujo de calor adicional en el generador, lo que hace que el desempeño de este sistema sea menor que el del sistema de compresión [3]. Este flujo de calor adicional puede provenir de fuentes de energía libre como la solar, o residuales, como el calor desechado, lo que puede ser ventajoso económicamente $[5,14]$. Los pares más comunes de fluidos son bromuro de litio/agua (refrigerante) o agua/amoníaco (refrigerante).

En el acondicionamiento de espacios, el par absorbente/refrigerante más utilizado es bromuro de litio/agua. Los sistemas de absorción de una etapa requieren una fuente externa de calor a una temperatura entre $80^{\circ} \mathrm{C}$ y $120{ }^{\circ} \mathrm{C}$, y tienen un desempeño menor. La ventaja de los sistemas de absorción versus los sistemas de compresión es la posibilidad de utilizar fuentes de energía libres o residuales $[5,14]$.

Finalmente, otra ventaja que ofrece este tipo de dispositivo en comparación con los equipos de compresión es su nivel de sonido suave y sin vibración.

\subsection{Descripción de los equipos actuales de ab- sorción}

\subsubsection{Equipo de agua/bromuro de litio}

El par agua/bromuro de litio es un fluido de absorción que ha sido utilizado ampliamente desde la década de 1950. Este fluido de trabajo utiliza el agua como refrigerante y está limitado a temperaturas de refrigeración por encima de $0{ }^{\circ} \mathrm{C}$. Las máquinas de absorción basadas en agua/bromuro de litio se configuran típicamente como equipos de agua para sistemas de aire acondicionado en edificios grandes, y están disponibles en tamaños de 35 a $5000 \mathrm{~kW}$.

El coeficiente de desempeño (COP) de estas máquinas, que se define como la capacidad de refrigeración dividida entre el calor de entrada, varía típicamente en el intervalo $0,7<\mathrm{COP}<1,2$ en función de la configuración del ciclo. La tecnología más utilizada son los equipos por compresión de vapor, y la selección entre estos equipos de compresión y absorción depende de factores económicos [5].

\subsubsection{Equipo de agua/amoníaco}

El amoníaco/agua es un par de fluidos de absorción que ha sido utilizado desde finales del siglo XIX para 
la producción de hielo, antes del desarrollo de la tecnología de compresión de vapor. El amoníaco es el refrigerante y el agua es el medio de transporte. Por lo tanto, el papel del agua es diferente en los pares amoníaco/agua y agua/bromuro de litio. Una ventaja del amoníaco como refrigerante es que permite trabajar con menores temperaturas porque el punto de congelación del amoníaco es $-77,7^{\circ} \mathrm{C}$. Sin embargo, la toxicidad del amoníaco es un factor que ha limitado su uso a áreas bien ventiladas.

El punto principal para la venta de los equipos con tecnología agua/amoníaco es su capacidad de proveer aire acondicionado a gas. Las máquinas están disponibles con una capacidad entre 10 y $90 \mathrm{~kW}$, con un COP entre 0,5 y 1,5. Estas unidades tienen un nicho en el mercado porque existen pocas tecnologías a gas apropiadas para múltiples aplicaciones. En particular, las aplicaciones industriales que son interesantes son las soluciones adaptadas a las necesidades del usuario que permiten utilizar calor de desecho o energía renovable $[5,12]$.

\subsection{Sistema de una etapa}

La Figura 2 muestra un sistema de absorción de una etapa (dos presiones de trabajo y una etapa de generación/absorción) que trabaja con amoníaco como refrigerante y agua como solvente. El amoníaco como refrigerante permite obtener menores temperaturas del evaporador en comparación con otras sustancias, y está disponible para una amplia variedad de aplicaciones. Por lo tanto, es uno de los refrigerantes clásicos $[5,11]$.

Los componentes de un sistema de refrigeración por absorción son:

- Evaporador: Remueve el calor del medio de enfriamiento en el equipo (solución de agua y glicol). El flujo de calor $\left(\dot{Q}_{\text {evap }}\right)$ que se remueve evapora el refrigerante (amoníaco- $\mathrm{NH}_{3}$ ) en un ambiente de baja temperatura y baja presión.

- Deflegmador: Es un intercambiador de calor donde ocurre la condensación parcial del vapor (rico en amoníaco) que viene del generador (1). El calor removido en este proceso permite obtener vapor puro de amoníaco que va hacia el condensador $(2 \mathrm{~V})$, y el líquido condensado (2L) (rico en agua) retorna al rectificador por gravedad.

- Condensador: En la entrada de este componente, el vapor de amoníaco está a alta temperatura, y luego se condensa cuando pasa a través del intercambiador de calor (3).

- Intercambiador de calor a gas: Este componente es un recuperador de calor, donde se transfiere calor del amoníaco líquido que viene del condensador (3) al vapor de amoníaco que va al absorbente. Esto ayuda a enfriar el refrigerante y aumentar el COP del sistema.

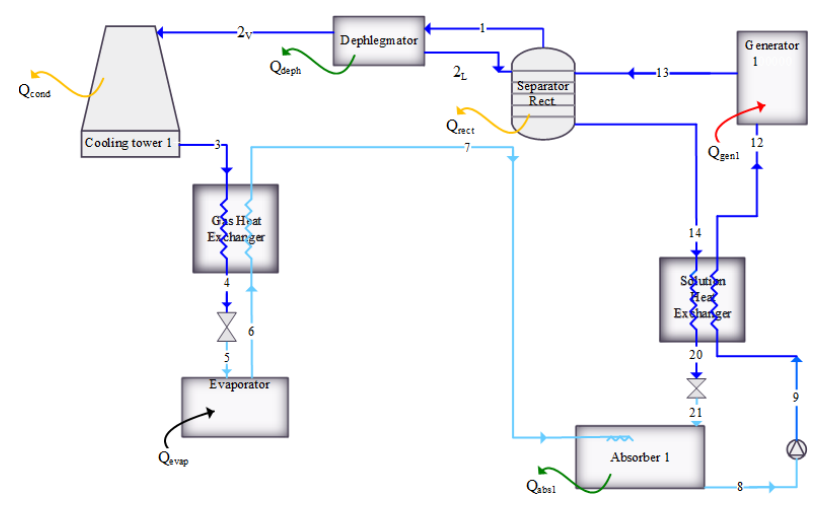

Figura 2. Sistema de absorción de una etapa.

- Válvulas de expansión: Reducen la presión del amoníaco líquido (4) y de la solución deficiente que va al absorbente (20).

- Absorbente: Una solución diluida de agua de amoníaco (21) absorbe el vapor refrigerante (7). La mezcla debe enfriarse simultáneamente para diluir suficiente amoníaco en la solución, obteniendo una solución rica a la salida de este componente (8). Por lo tanto, para cumplir con este proceso es necesario remover un flujo $\left(\dot{Q}_{a b s}\right)$ de calor.

- Bomba: Transporta la solución rica (8), incrementando la presión del fluido que es una solución incompresible (líquida).

- Intercambiador de calor de la solución: Este componente es un recuperador de calor, donde la solución deficiente (14) transfiere calor a la solución rica (9) para ser precalentada.

- Generador: En este componente se calienta la solución rica (12), para obtener vapor con una alta concentración de amoníaco (13 V), y la solución es diluida con respecto al amoníaco.

- Rectificador: Es una columna de fraccionamiento requerida para condensar el agua parcialmente vaporizada que sale del generador, y producir únicamente vapor de amoníaco de alta pureza (1) que va hacia el deflegmador.

El COP de un sistema de una etapa es la relación entre el flujo de calor removido en el evaporador respecto al flujo de calor que entra al generador y a la potencia consumida por la bomba, es decir:

$$
C O P=\frac{\dot{Q}_{e v a p}}{\dot{Q}_{g e n 1}+\dot{W}_{b o m b 1}}
$$


donde:

COP - coeficiente de desempeño

$\dot{Q}_{\text {evap }}$ - flujo de calor en el evaporador $(\mathrm{kW})$

$\dot{Q}_{g e n 1}$ - flujo de calor en el generador $1(\mathrm{~kW})$

$\dot{W}_{\text {bomb1 }}$ potencia mecánica de la bomba $1(\mathrm{~kW})$

\subsection{Sistema de dos etapas (equipo AGO)}

La Figura 3 muestra el esquema del sistema estudiado en este proyecto, y la información fue obtenida de un diagrama unifilar de instalación del proyecto AGO [11]. La figura muestra los componentes principales del sistema de refrigeración y de los sistemas auxiliares.

El sistema de refrigeración estudiado no es el ciclo de absorción de dos etapas y que es comúnmente descrito en la literatura $[1,5]$. Este tiene dos etapas de procesos de generación y absorción, donde el vapor rico en amoníaco sale únicamente del generador 1 (13) al deflegmador; y la solución líquida sale del rectificador (14) y va al generador 2, donde se le agrega calor a la solución. Como resultado, después del separador 2, el estado (17) absorbe mayor cantidad de amoníaco. Asimismo, se obtiene una solución deficiente (20) que va al absorbente 2 y al absorbente 1 , respectivamente.
El objetivo principal de este equipo de dos etapas es recuperar más energía del agua caliente para suministrarla a los fluidos de trabajo del sistema de refrigeración. En este proyecto, se realiza una comparación de modelos termodinámicos para un sistema de refrigeración por absorción de una etapa (Figura 2) y un sistema de doble etapa (Figura 3).

El COP de un sistema de doble etapa está dado por la relación entre el flujo de calor removido en el evaporador respecto al calor consumido en los generadores y la potencia mecánica requerida por las bombas, Ecuación (2), es decir:

$$
C O P=\frac{\dot{Q}_{e v a p}}{\dot{Q}_{g e n 1}+\dot{Q}_{g e n 2}+\dot{W}_{b o m b a 1}+\dot{W}_{b o m b a 2}}
$$

donde:

COP - coeficiente de desempeño

$\dot{Q}_{\text {evap }}$ - flujo de calor en el evaporador $(\mathrm{kW})$

$\dot{Q}_{g e n 1}$ - flujo de calor en el generador $1(\mathrm{~kW})$

$\dot{Q}_{g e n 2}$ - flujo de calor en el generador $2(\mathrm{~kW})$

$\dot{W}_{\text {bomba1 }}$ - potencia mecánica de la bomba $1(\mathrm{~kW})$

$\dot{W}_{\text {bomba2 }}$ - potencia mecánica de la bomba $2(\mathrm{~kW})$

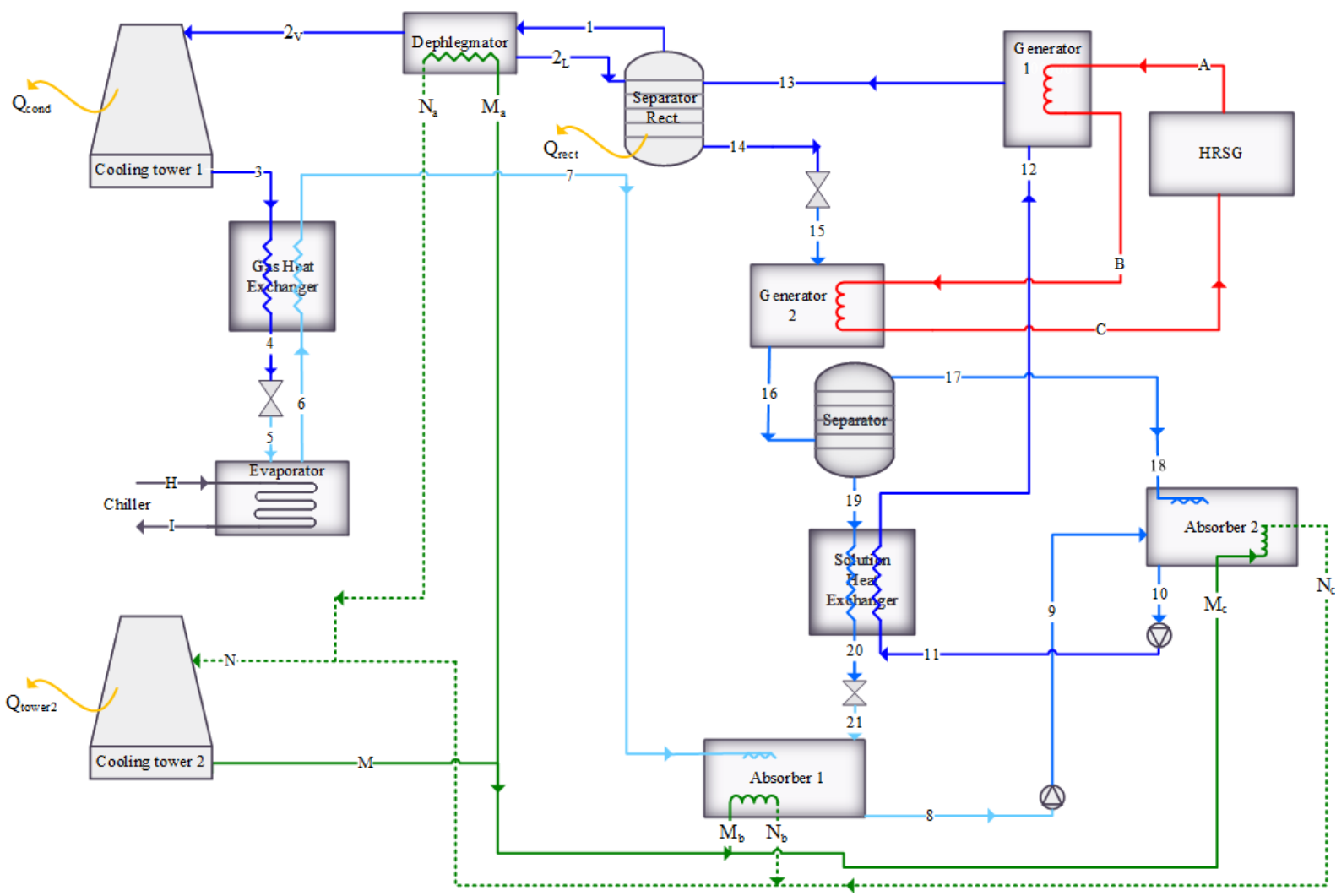

Figura 3. Elementos necesarios para la toma de muestras. 


\subsection{Sistemas auxiliares}

En la Figura 3 se muestran los sistemas auxiliares que se describen a continuación y que interactúan con diferentes componentes del sistema de absorción:

- HRHWG: Es un intercambiador de calor que utiliza la energía desechada por el motor para producir agua caliente. En este caso, el agua caliente (A) a $110{ }^{\circ} \mathrm{C}$ entra en el generador 1 , entrega calor a la solución amoníaco/agua y sale por (B) a $86{ }^{\circ} \mathrm{C}$. Esta agua continúa hacia el generador 2 donde intercambia calor con la solución, y sale por (C) a $80{ }^{\circ} \mathrm{C}$. En condiciones normales de operación, el flujo volumétrico de agua es $120 \mathrm{~m}^{3} / \mathrm{h}$.

- Evaporador del chiller: El calor absorbido por el refrigerante $\left(\mathrm{NH}_{3}\right)$ en el evaporador se remueve de una solución (glicol-agua). En este sistema, el glicol entra en $(\mathrm{H})$ a $-2{ }^{\circ} \mathrm{y}$ sale por (I) a $-8{ }^{\circ} \mathrm{C}$. La simulación realizada en este proyecto ayuda a determinar el flujo másico de solución de glicol que puede ser enfriada por este equipo.

- Torre de enfriamiento 1: En el condensador el refrigerante necesita expulsar calor, lo cual ocurre en esta torre enfriada por agua y aire, con una capacidad de $1250 \mathrm{~kW}$.

- Torre de enfriamiento 2: El calor expulsado en el absorbente 1 , absorbente 2 y deflegmador, evapora el amoníaco (M) y ese vapor va a la torre de enfriamiento 2 para condensarse y continuar el proceso. La capacidad de esta torre es de $3750 \mathrm{~kW}$.

El flujo de calor que se intercambia con el sistema de refrigeración en el HRHWG y en el equipo evaporador se calcula directamente usando la Ecuación (3).

$$
\dot{Q}=\dot{m} \times c_{p} \times \Delta T
$$

donde:

$\dot{Q}$ - flujo de calor en el intercambiador (kW)

$\dot{m}$ - flujo másico de agua $(\mathrm{kg} / \mathrm{s})$

$C_{p}$ - calor específico del agua $\left(\mathrm{kJ} \cdot \mathrm{kg} /{ }^{\circ} \mathrm{C}\right)$

$\Delta T$ - diferencia de temperatura $\left({ }^{\circ} \mathrm{C}\right)$

El flujo de calor removido por las torres de enfriamiento son datos de entrada para el modelo del sistema de refrigeración desarrollado en este proyecto. Las capacidades caloríficas de las torres de enfriamiento se utilizan como parámetros de este modelo, y los procesos termodinámicos y de transferencia de calor pueden analizarse detalladamente en trabajos futuros.

\section{Desarrollo del modelo}

\subsection{Análisis termodinámico}

El modelo fue desarrollado usando EES (Engineering Equation Solver) [15], debido a que este software tiene una rutina externa para conocer las propiedades de la solución amoníaco-agua y de la mezcla de gases. Para realizar el estudio termodinámico de los sistemas presentados en la Figura 2 y en la Figura 3, se desarrolló una metodología apropiada para cada componente, tal como se explica a continuación:

- Generador: La masa total, masa de amoníaco y el balance de energía, se expresan respectivamente con las Ecuaciones (4), (5) y (6).

$$
\begin{gathered}
\dot{m}_{12}=\dot{m}_{13 V}+\dot{m}_{13 L} \\
\dot{m}_{12} \times x_{12}=\dot{m}_{13 V} \times x_{13 V}+\dot{m}_{13 L} \times x_{13 V} \\
\dot{m}_{12} \times h_{12}+\dot{Q}_{g e n 1}=\dot{m}_{13 V} \times h_{13 V}+\dot{m}_{13 L} \times h_{13 V}
\end{gathered}
$$

donde:

$\dot{m}_{12}$ - flujo másico de la mezcla de gases $(\mathrm{kg} / \mathrm{s})$

$\dot{m}_{13 V}$ - flujo másico de la mezcla de gases $(\mathrm{kg} / \mathrm{s})$

$\dot{m}_{13 L}$ - flujo másico de la solución (líquido) $(\mathrm{kg} / \mathrm{s})$

$x_{12}$ - fracción de la masa de amoníaco en el generador 1

$x_{13 V}$ - fracción de la masa del vapor de amoníaco

$h_{12}$ - entalpía específica de la mezcla de gases en el generador $1(\mathrm{~kJ} / \mathrm{kg})$

$\dot{Q}_{g e n 1}$ - flujo de calor del generador $1(\mathrm{~kW})$

$h_{13 V}$ - entalpía específica del amoníaco en el generador $1(\mathrm{~kJ} / \mathrm{kg})$

A partir de esta sección es importante notar que la cantidad de calor suministrada a la solución aguaamoníaco que fluye a través del generador es igual al calor entregado por el HRHWG definido por la Ecuación (7).

$$
\dot{Q} g e n 1_{H R H W G}=\dot{m}_{A} \times c_{p} \times\left(T_{A}-T_{B}\right)
$$

donde:

$\dot{Q} g e n 1_{H R H W G}$ - flujo de calor en el HRHWG (kW) $\dot{m}_{A}$ - flujo másico de agua (HRHWG) $(\mathrm{kg} / \mathrm{s})$

$C_{p}$ - calor específico del agua $\left(\mathrm{kJ} * \mathrm{~kg} /{ }^{\circ} \mathrm{C}\right)$

$T_{A}$ - temperatura de entrada al generador $1\left({ }^{\circ} \mathrm{C}\right)$

$T_{B}$ - temperatura de salida del generador $1\left({ }^{\circ} \mathrm{C}\right)$

Los balances de masa y energía para el generador 2 se realizaron bajo los mismos criterios. 
- Rectificador: Se realizaron balances de masa y energía, con la particularidad que el rectificador disipa calor al ambiente como respuesta a su participación en la condensación de la solución vaporizada de amoníaco-agua que viene del generador. Este elemento lleva a cabo la separación de las fases líquida y gaseosa de la solución amoníaco que fluye a través de él. El rectificador es una columna de fraccionamiento necesaria para condensar el agua parcialmente vaporizada que sale del generador, y obtener únicamente amoníaco-vapor de muy alta pureza (1) que va al deflegmador.

- Deflegmador: El calor se transfiere al sistema auxiliar de amoníaco, para asegurar que el refrigerante retorna como vapor a la torre de enfriamiento 2. El flujo de calor removido está dado por la Ecuación (8).

$$
\dot{Q}_{d e f}=\dot{m}_{M a} \times\left(h_{N a}-h_{M a}\right)
$$

donde:

$\dot{Q}_{\text {deph }}$ - flujo de calor al deflegmador (kW)

$\dot{m}_{M a}$ - flujo másico de amoníaco en el deflegmador $(\mathrm{kg} / \mathrm{s})$

$h_{N a}$ - entalpía específica del amoníaco que sale $(\mathrm{kJ} / \mathrm{kg})$

$h_{M a}$ - entalpía específica del amoníaco que entra $(\mathrm{kJ} / \mathrm{kg})$

- Condensador: el calor removido del vapor de amoníaco a la entrada es liberado hacia la atmósfera en la torre de enfriamiento.

- Intercambiador de calor a gas: En el balance de energía se determinan primero las capacidades caloríficas de los fluidos que entran al intercambiador de calor utilizando las propiedades caloríficas específicas del amoníaco, para luego obtener el calor recuperado por el vapor de amoníaco que va al absorbente 1 . Luego se identifica la capacidad calorífica mínima, así como la mayor diferencia de temperatura. De esta manera, el máximo flujo de calor que puede transferirse será igual al producto de la capacidad calorífica mínima y la mayor diferencia de temperatura. Finalmente, la efectividad del intercambiador de calor está dada por la Ecuación (9).

$$
\varepsilon=\frac{\dot{Q}^{r e c u p}}{G H X}
$$

donde:

$\varepsilon$ - efectividad del intercambiador de calor

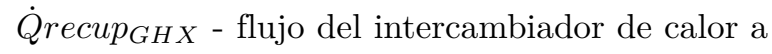
gas $(\mathrm{kW})$

$\dot{Q}_{\max }$ - máximo flujo de calor del GHX (kW)

El balance de energía para el intercambiador de calor de solución se realizó bajo los mismos criterios, con la única diferencia que el intercambio de calor en el SHX ocurre de la solución rica a la solución deficiente.

- Válvulas de expansión: Para estos componentes se suponen flujo másico y entalpía constante.

- Evaporador: El calor que pierde la mezcla de glicol-agua (medio de enfriamiento) es igual al calor ganado por el refrigerante (amoníaco) que circula a través del evaporador. El flujo de calor en el evaporador se calcula con la Ecuación (10).

$$
\dot{Q}_{\text {evap }}=\dot{m}_{\text {glicol }} \times c_{p-\text { propileno }} \times\left(T_{H}-T_{I}\right)
$$

donde:

$\dot{Q}_{\text {evap }}$ - flujo de calor del equipo (kW)

$\dot{m}_{\text {glicol }}$ - flujo másico de glicol $(\mathrm{kg} / \mathrm{s})$

$T_{H}$ - temperatura a la entrada del equipo $\left({ }^{\circ} \mathrm{C}\right)$

$T_{I}$ - temperatura a la salida del equipo $\left({ }^{\circ} \mathrm{C}\right)$

$C_{p \text {-propileno }}$ - calor específico de la mezcla glicolagua $\left(\mathrm{kJ} \cdot \mathrm{kg} /{ }^{\circ} \mathrm{C}\right)$

- Absorbente: El calor perdido por la mezcla amoníaco-agua en el absorbente 1 es tomado por el sistema de refrigeración auxiliar mencionado en la sección correspondiente al deflegmador, y es el mismo proceso en el absorbente 2. El flujo de calor perdido en el absorbente está dado por la Ecuación (11).

$$
\dot{Q}_{a b s 1}=\dot{m}_{M b} \times\left(h_{N b}-h_{M b}\right)
$$

donde:

$\dot{Q}_{a b s 1}$ - flujo de calor en el absorbente $1(\mathrm{~kW})$

$\dot{m}_{M b}$ - flujo másico de amoníaco en el absorbente $1(\mathrm{~kg} / \mathrm{s})$

$h_{N b}$ - entalpía específica del amoníaco en la salida del absorbente $1(\mathrm{~kJ} / \mathrm{kg})$

$h_{M b}$ - entalpía específica del amoníaco en la entrada del absorbente $1(\mathrm{~kJ} / \mathrm{kg})$

El deflegmador y ambos absorbentes proporcionan el calor utilizado por el sistema de refrigeración auxiliar. 
- Bomba: Para calcular la energía requerida por la bomba se inicia con la fórmula que describe la potencia absorbida por una bomba, considerando el volumen específico y la diferencia de presión. Esto es igual al trabajo de la bomba como un cambio de entalpía, Ecuación (12).

$$
\dot{W}_{b o m b a 1}=\dot{m}_{8} \times\left(h_{9}-h_{8}\right)
$$

donde:

$\dot{W}_{\text {bomba1 - potencia mecánica de la bomba }(\mathrm{kW})}$

$\dot{m}_{8}$ - flujo másico de la solución $(\mathrm{kg} / \mathrm{s})$

$h_{9}$ - entalpía específica de la solución en la salida de la bomba $1(\mathrm{~kJ} / \mathrm{kg})$

$h_{8}$ - entalpía específica de la solución en la entrada de la bomba $1(\mathrm{~kJ} / \mathrm{kg})$

El sistema de refrigeración analizado presenta dos etapas de absorción y generación, que difieren del ciclo comúnmente utilizado por la industria (de una etapa). Por esta razón, se simularon dos tipos de ciclos en EES [15] con las mismas condiciones operacionales como data de entrada.

\subsection{Data de entrada al modelo}

La data de entrada fue definida basándose en las condiciones operacionales de diseño. La alta presión del equipo es $1,700 \mathrm{kPa}$, la temperatura del amoníaco en el evaporador es $-12{ }^{\circ} \mathrm{C}$. En consecuencia, la baja presión corresponde a la presión de saturación $(267,9 \mathrm{kPa})$.

El HRHWG entrega $120 \mathrm{~m}^{3} / \mathrm{h}$ de agua a los generadores a $108{ }^{\circ} \mathrm{C}$, y el sistema de generación baja la temperatura del agua a $80^{\circ} \mathrm{C}$. En el evaporador, la temperatura del refrigerante del equipo (agua y glicol) se reduce en $6{ }^{\circ} \mathrm{C}$.

El análisis termodinámico se ha realizado enfocado en la solución amoníaco-agua y en la concentración de la mezcla de gases. Los valores comunes de concentración para una solución rica están entre 0,35 y $0,45[14,16]$. La concentración de la solución en la entrada del generador 2 corresponde a una concentración media $\left(x_{\text {media }}\right)$, que ha sido definida como una función de la capacidad de las torres de enfriamiento $(1,250 \mathrm{~kW}$ and $3,750 \mathrm{~kW})$. La concentración media es 0,36 , como se muestra en la Figura 4.

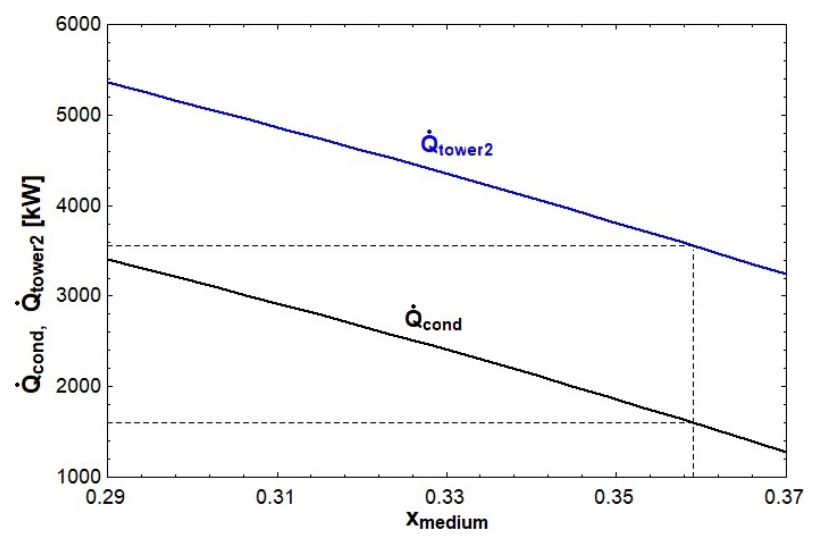

Figura 4. Flujo de calor del condensador y la torre de enfriamiento 2 vs. concentración media de la solución.

\subsection{Factor de utilización de la PCP}

El factor de utilización de una planta de cogeneración es el cociente de las potencias de salida (energías térmica y eléctrica) con respecto al calor total que entra a la PCP, Ecuación (13).

$$
\epsilon_{u}=\frac{\dot{Q}_{g e n 1}+\dot{Q}_{g e n 2}+\dot{W}_{\text {neto }}}{\dot{Q}_{\text {entrada }}}
$$

donde:

$\epsilon_{u}$ - factor de utilización de la PCP

$\dot{Q}_{g e n 1}$ - flujo de calor del generador $1(\mathrm{~kW})$

$\dot{Q}_{g e n} 2$ - flujo de calor del generador $2(\mathrm{~kW})$

$\dot{Q}_{\text {entrada }}$ - calor total que entra a la PCP $(\mathrm{kW})$

$\dot{Q}_{g e n}$ es la energía térmica recuperada para ser utilizada como calor de proceso en el equipo. $\dot{W}_{\text {neto }}$ es la potencia neta obtenida en las turbinas de gas y de vapor, y $\dot{Q}_{\text {entrada }}$ es el calor total que entra, que corresponde a la energía calorífica consumida por los motores de combustión interna.

\subsection{Ahorro económico con respecto a un sis- tema de refrigeración por compresión de vapor}

La reducción del consumo de potencia eléctrica con el SRA respecto a un sistema de refrigeración por compresión de vapor permite obtener ahorros económicos. Estos ahorros han sido calculados comparando con un sistema de refrigeración por compresión de vapor de la misma capacidad de enfriamiento.

El sistema de enfriamiento trabaja las 24 horas del día, pero de acuerdo con sus características principales puede suponerse que el factor de utilización es menor que uno porque el compresor empieza a trabajar justo cuando la temperatura se eleva por encima de cierto límite; por lo tanto, como data de campo experimental, puede ajustarse a $50 \%$. 


\section{Resultados y análisis}

\subsection{Resultados del modelo}

El ciclo de dos etapas permite recuperar de mejor manera la energía del agua HRHWG. Por esta razón, la temperatura de la solución en la entrada del generador principal se incrementa, y es posible obtener un flujo de solución más rica con el mismo calor disponible del agua.

Tabla 1. Comparación de los ciclos de una etapa vs. dos etapas

\begin{tabular}{cccc}
\hline & Unidades & $\begin{array}{c}\text { Dos } \\
\text { etapas }\end{array}$ & $\begin{array}{c}\text { Una } \\
\text { etapa }\end{array}$ \\
\hline $\begin{array}{c}\text { Calor recuperado } \\
\text { en el GEN 1 }\end{array}$ & {$[\mathrm{kW}]$} & 2,954 & 2,954 \\
$\begin{array}{c}\text { Calor recuperado } \\
\text { en el GEN 2 }\end{array}$ & {$[\mathrm{kW}]$} & 800 & - \\
$\begin{array}{c}\text { Temperatura } \\
\text { de la solución }\end{array}$ & & & \\
$\begin{array}{c}\text { a la entrada } \\
\text { del generator 1 }\end{array}$ & {$\left[{ }^{\circ} \mathrm{C}\right]$} & 75 & 65 \\
Capacidad evaporador & {$[\mathrm{kW}]$} & 1,6 & 1,3 \\
COP & - & 0.44 & 0.44 \\
\hline
\end{tabular}

Tal como se muestra en la Tabla 1, al agregar una segunda etapa de absorción y generación al ciclo de absorción de una etapa, se incrementa en un $23 \%$ la capacidad de refrigeración. El COP es similar para ambos sistemas (una y dos etapas), pero si se considera que el calor que entra al sistema de refrigeración de dos etapas fue recuperado de energía desechada, es más ventajoso incluir una etapa adicional de la generación y absorción para incrementar la capacidad de enfriamiento.

La concentración de la solución a la entrada del generador 2, es la variable que más influye en el modelo. Cuando presenta valores menores a 0,36, el COP del sistema se incrementa debido a que aumenta el flujo de amoníaco proveniente del generador 2, tal como se muestra en la Figura 5. En consecuencia, la capacidad del evaporador mejora, manteniendo constante el calor requerido por los generadores de amoníaco.

La disponibilidad de calor es un aspecto fundamental, y depende de las condiciones de operación del motor. Si el motor está trabajando a la máxima carga (máxima capacidad de HRSG y HRHWG), el generador 2 produce un mayor flujo de amoníaco, y el sistema de refrigeración alcanza su capacidad máxima $(1,600 \mathrm{~kW})$, con un COP de 0.43. El COP se mantiene constante a pesar de que el calor recuperado disminuye y la capacidad del evaporador se reduce como se muestra en la Figura 6.

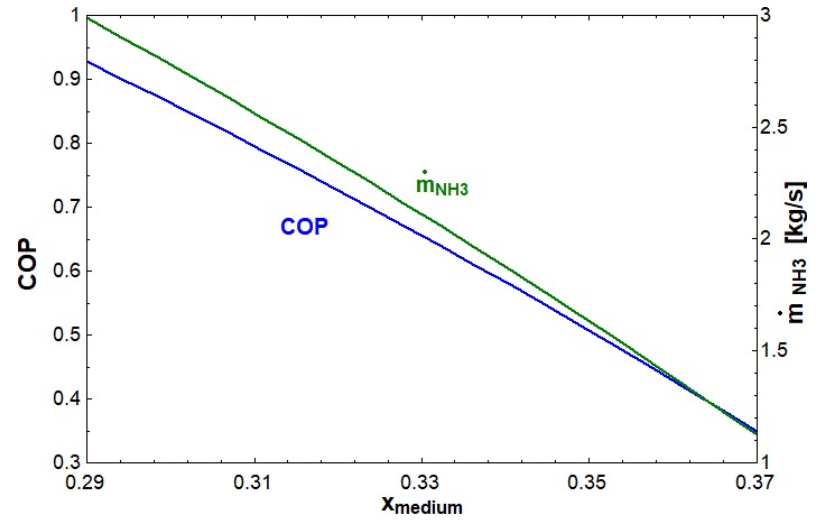

Figura 5. COP y flujo másico de refrigerante respecto a la concentración media de la solución.

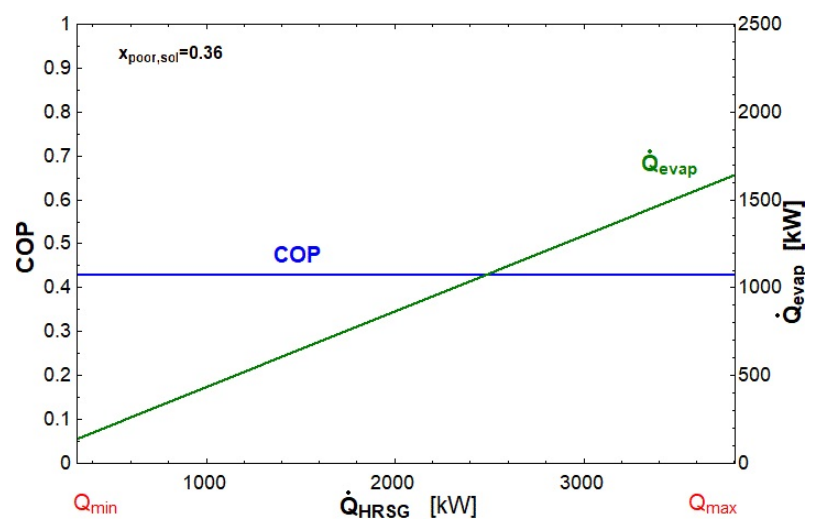

Figura 6. COP y flujo de calor en el evaporador respecto al calor recuperado en la PCP.

La presión alta del sistema es una condición de operación esencial, y está definida por la geografía donde el equipo está instalado, debido a la altitud. Si el sistema de absorción opera a nivel del mar, la capacidad del evaporador se incrementa y mejora el COP porque el calor requerido por los generadores de amoníaco permanece constante, tal como se muestra en la Figura 7. Si la presión alta sube, aumenta la capacidad de la torre de enfriamiento (requerida para disipar calor de los separadores, absorbentes y deflegmador). Por esta razón, la capacidad de la torre de enfriamiento debe diseñarse, según las condiciones operacionales in situ.

La efectividad de los intercambiadores de calor fue considerada constante e igual a 0,8 [17]. El intercambiador de calor de solución permite recuperar calor dentro del ciclo, e incrementa la temperatura de la solución amoníaco-agua antes de que entre al generador principal. La efectividad del SHX es directamente proporcional al flujo de calor del evaporador y al COP del sistema, como puede verse en la Figura 8. Un SHX de mayor efectividad representa un alto costo de inversión, y debe ser evaluado considerando que el COP y la capacidad de enfriamiento también se incrementan. 


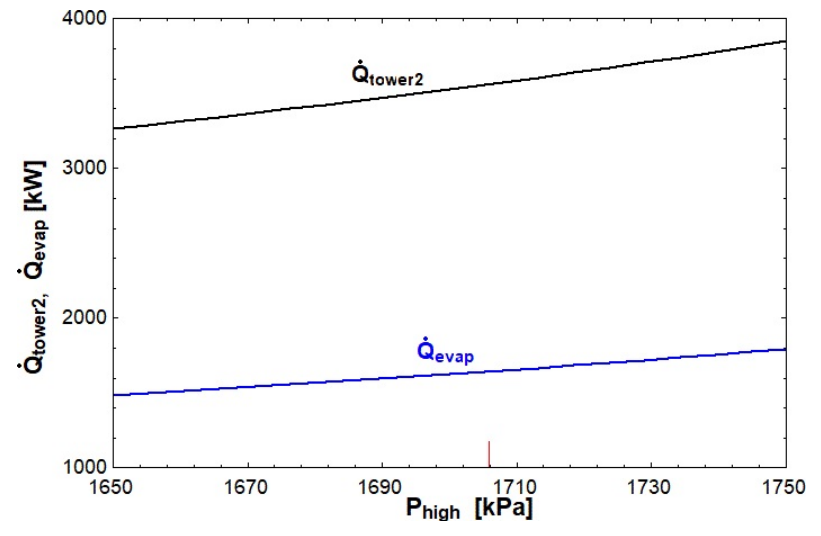

Figura 7. Flujo de calor en la torre de enfriamiento 2 y en el evaporador respecto a la presión alta del sistema.

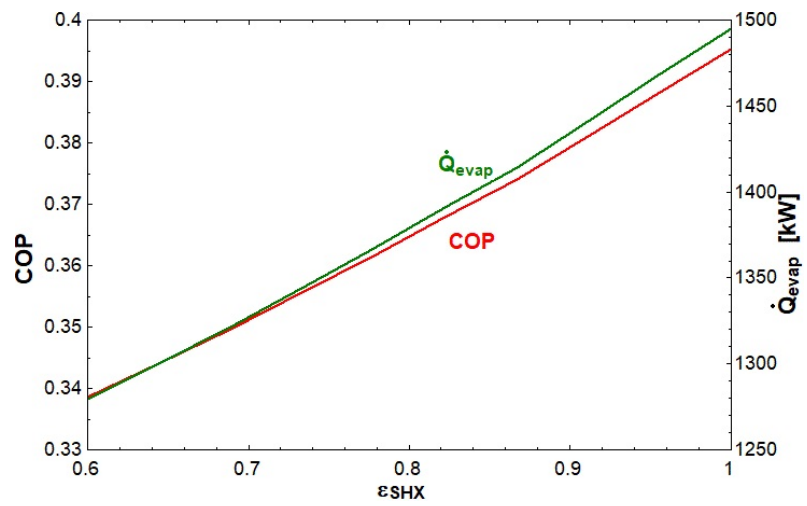

Figura 8. COP y flujo de calor en el evaporador respecto a la efectividad del SHX.

\subsection{Factor de utilización de la PCP}

$\dot{Q}_{g e n}$ es el calor recuperado para usar la energía desechada en el equipo, y es igual a 3,8 MW (t) (obtenida por el modelo). El $\dot{W}_{\text {neto }}$ es $28,1 \mathrm{MW}$ (e) y corresponde a la potencia neta de los motores de combustión interna (8,7 MW (e) por motor) y de la turbina de vapor $(2 \mathrm{MW}(\mathrm{e})) \cdot \dot{Q}_{\text {entrada }}$ se calcula como muestra la Ecuación (14), con la información técnica del fabricante de los motores Roll Royce, que reporta $7,572 \mathrm{~kJ} / \mathrm{kWh}$ de consumo específico de energía. El factor de utilización para el proceso se calcula con la Ecuación (13) y el resultado se muestra en la Ecuación (15).

$$
\begin{gathered}
\dot{Q}_{\text {entrada }}=3 \times 8,700 k w(e)\left(\frac{7,572 k J(f)}{1 k W h(e)}\right) \times \frac{1 h}{3600 s} \\
\dot{Q}_{\text {entrada }}=54,897 k W(f)
\end{gathered}
$$

$$
\epsilon_{u}=\frac{3,8 M W+(2+8,7 \times 3) M W}{54,897 M W}=0,5811
$$

El flujo de calor que entra a la PCP es kW(f), donde f representa la energía del combustible.

El factor de utilización del proceso de cogeneración es 58,11\%, lo cual significa que el proceso está utilizando un alto porcentaje de la energía del combustible consumido por los motores de combustión interna.

\subsection{Estimación del ahorro económico}

La capacidad de enfriamiento del sistema de absorción refrigeración (AGO) de dos etapas fue calculada con el modelo, resultando un valor de 1,623 kW (t). Para conocer el ahorro económico, en esta sección se calcula la electricidad que consumiría un equipo común de compresión de vapor.

El mercado ofrece muchos tipos de máquinas que utilizan el ciclo de compresión de vapor para refrigerar. El COP promedio es 4,5 [18]. Por lo tanto, es posible calcular la potencia mecánica $\dot{W}_{\text {comp }}$ requerida por el compresor dada por la Ecuación (16).

$$
\dot{W}_{c o m p}=\frac{\dot{Q}_{e v a p}}{C O P}=\frac{1,623 k W}{4,5}=360,67 k W(e)
$$

El factor de utilización de un equipo de compresión fue definido como $50 \%$ en la sección 3.4. Si se consideran 24 horas al día y 365 días por año, el consumo anual de electricidad del sistema de compresión es 1579 734,60 kWh/año.

Considerando un costo promedio unitario de la energía (obtenido del costo mensual de electricidad de la tarifa industrial para el último año en Agua Prieta/México [19]) de 0,09 USD/kWh, el costo de electricidad evitado por el sistema de cogeneración sería 142 000,00 USD/año, aproximadamente.

Sin embargo, si se consideran los posibles ahorros y se fija un tiempo de 5 años para recuperar el dinero, la inversión inicial podría estar alrededor de 710 000,00 USD. Esto significa que la inversión es rentable. Para obtener resultados más precisos, es necesario adquirir data específica.

La cogeneración eficiente tiene múltiples incentivos fiscales y preferencia en el despacho de energía en diferentes países (por ejemplo, México), debido a la contribución de la tecnología en la preservación del ambiente.

\section{Conclusiones}

Es conveniente utilizar un sistema de absorción de dos etapas, debido a que permiten explotar mejor el calor disipado por los motores. Asimismo, los COP de ambos sistemas (de una y de dos etapas) son similares.

La concentración de la solución a la salida del generador 1 es la variable con más influencia en los resultados del ciclo. Por esta razón, esta variable fue 
elegida de acuerdo con la capacidad de las torres de enfriamiento instaladas. Además, se observó que si la concentración está cercana a 0,3, el COP del sistema está cercano a 1 mientras que se logran mayores valores de concentración.

El COP se mantiene constante, independientemente de la cantidad de flujo de calor que entra al sistema. Si se le suministra al generador una mayor cantidad de calor, aumenta el flujo másico de amoníaco que va al evaporador, y consecuentemente la capacidad de refrigeración del equipo se incrementa.

El proceso de cogeneración utilizando gases de escape como fuente de calor para un equipo de refrigeración por absorción puede proporcionar conservación del ambiente y beneficios económicos.

\section{Referencias}

[1] ASHRAE, Handbook - Fundamentals. 2009, inch-pound ed., American Society of Heating, Refrigerating and Air-Conditioning Engineers, Inc., 2009. [Online]. Available: https://goo.gl/X11TGj

[2] C. Moné, D. Chau, and P. Phelan, "Economic feasibility of combined heat and power and absorption refrigeration with commercially available gas turbines," Energy Conversion and Management, vol. 42, no. 13, pp. 1559-1573, 2001. [Online]. Available: https://doi.org/10.1016/S0196-8904(00)00157-6

[3] J. Rodríguez-Muñoz and J. Belman-Flores, "Review of diffusion-absorption refrigeration technologies," Renewable and Sustainable Energy Reviews, vol. 30, pp. 145-153, 2014. [Online]. Available: https://doi.org/10.1016/j.rser.2013.09.019

[4] K. Ullah, R. Saidur, H. Ping, R. Akikur, and N. Shuvo, "A review of solar thermal refrigeration and cooling methods," $R e$ newable and Sustainable Energy Reviews, vol. 24, pp. 499-513, 2013. [Online]. Available: https://doi.org/10.1016/j.rser.2013.03.024

[5] K. E. Herold, R. Radermacher, and S. A. Klein, Absorption Chillers and Heat Pumps. CRC Press, 2016, ch. 10. Two-Stage Ammonia/Water Systems, pp. 215-232. [Online]. Available: https://goo.gl/MrMG2m

[6] D. Colorado and W. Rivera, "Performance comparison between a conventional vapor compression and compression-absorption single-stage and double-stage systems used for refrigeration," Applied Thermal Engineering, vol. 87, pp. 273-285, 2015. [Online]. Available: https: //doi.org/10.1016/j.applthermaleng.2015.05.029
[7] M. Conde, Thermophysical properties of $\mathrm{NH}_{3}+$ $\mathrm{H}_{2} \mathrm{O}$ Solutions for the industrial design of absorption refrigeration equipment, Formulation for industrial use. M. Conde Engineering. p. 11. 2004.

[8] S. Said, K. Spindler, M. El-Shaarawi, M. Siddiqui, F. Schmid, B. Bierling, and M. Khan, "Design, construction and operation of a solar powered ammonia-water absorption refrigeration system in saudi arabia," International Journal of Refrigeration, vol. 62, pp. 222-231, 2016. [Online]. Available: https://doi.org/10.1016/j.ijrefrig.2015.10.026

[9] Y. Wang, C. Wang, and X. Feng, "Optimal match between heat source and absorption refrigeration," Computers \& Chemical Engineering, vol. 102, pp. 268-277, 2017. [Online]. Available: https: //doi.org/10.1016/j.compchemeng.2016.11.003

[10] S. Du, R. Wang, and X. Chen, "Development and experimental study of an ammonia water absorption refrigeration prototype driven by diesel engine exhaust heat," Energy, vol. 130, pp. 420-432, 2017. [Online]. Available: https://doi.org/10.1016/j.energy.2017.05.006

[11] AGO AG. (2017) Cooling from waste heat: efficient energy supply - from small to large-scale industrial projects. AGO AG Energie + Anlage. [Online]. Available: https://goo.gl/id5TYk

[12] V. Chakravarthy, R. Shah, and G. Venkatarathnam, "A review of refrigeration methods in the temperature range 4-300 k." ASME Journal of Thermal Science and Engineering Applications, vol. 3, no. 2, pp. 020 801-020 819, 2011. [Online]. Available: http://doi.org/10.1115/1.4003701

[13] R. J. Dossat, Principios de Refrigeración. Compañía Editorial Continental, 1980. [Online]. Available: https://goo.gl/CG3Tbg

[14] P. Srikhirin, S. Aphornratana, and S. Chungpaibulpatana, "A review of absorption refrigeration technologies," Renewable and Sustainable Energy Reviews, vol. 5, no. 4, pp. 343-372, 2001. [Online]. Available: https://doi.org/10.1016/S1364-0321(01)00003-X

[15] F-chart software. (2016) Engineering Equation Solver (EES). [Online]. Available: https://goo.gl/sYqcRc

[16] Y. Fan, L. Luo, and B. Souyri, "Review of solar sorption refrigeration technologies: Development and applications," Renewable and Sustainable Energy Reviews, vol. 11, no. 8, pp. 1758-1775, 2007. [Online]. Available: https://doi.org/10.1016/j.rser.2006.01.007 
[17] S. Steiu, D. Salavera, J. C. Bruno, and A. Coronas, "A basis for the development of new ammonia-water-sodium hydroxide absorption chillers," International Journal of Refrigeration, vol. 32, no. 4, pp. 577-587, 2009. [Online]. Available: https://doi.org/10.1016/j.ijrefrig.2009.02.017
[18] F. Kreith, S. Wang, and P. Norton, Air conditioning and refrigeration engineering. CRC Press, 1999. [Online]. Available: https://goo.gl/675PxJ

[19] SENER. (2017) Precios medios de energía eléctrica por tipo de tarifa. Secretaría de Energía. México. [Online]. Available: https://goo.gl/sz22NF 